\title{
ANITA (Advanced Network for Isotope and TArget Laboratories) - the Urgent Need for a European Target Preparation Network
}

\author{
Dorothea Schumann $^{\mathrm{a}}$, Goedele Sibbens ${ }^{\mathrm{b}}$, Anna Stolarz ${ }^{\mathrm{c}}$, Klaus Eberhardt ${ }^{\mathrm{d}}$, Bettina \\ Lommel $^{\mathrm{e}}$, Christelle Stodel ${ }^{\mathrm{f}}$
}

\author{
aPaul Scherrer Institute, 5232 Villigen PSI, Switzerland \\ ${ }^{b}$ European Commission, Joint Research Centre, Directorate G, Geel, Belgium \\ 'University of Warsaw, Poland \\ dJohannes Gutenberg-Universität and Helmholtz Institute Mainz, Germany \\ ${ }^{\mathrm{e}} \mathrm{Helmholtz}$ Centre for Heavy Ion Research Darmstadt, Germany \\ ${ }_{\mathrm{f}}^{\mathrm{f}}$ rand Accélérateur National d’Ions Lourds; CNRS/IN2P3-CEA/DRF Bvd Henri Becquerel F-14076 Caen, France
}

${ }^{a)}$ Corresponding author: dorothea.schumann@psi.ch

\begin{abstract}
A wide number of research fields in the nuclear sector requires high-quality and well-characterized samples and targets. Currently, only a few laboratories own or have access to the equipment allowing fulfilling such demands. Coordination of activities and sharing resources is therefore mandatory to meet the increasing needs. This very urgent issue has now been addressed by six European target laboratories with an initiative called ANITA (Advanced Network for Isotope and TArget laboratories). The global aim of ANITA is to establish an overarching research infrastructure service for isotope and target production and develop a tight cooperation between the target laboratories in Europe in order to transfer the knowledge and improve the production techniques of well-characterized samples and targets. Moreover, the interaction of the target producers with the users shall be encouraged and intensified to deliver tailor-made targets best-suited to the envisaged experiments. For the realization of this ambitious goal, efforts within the European Commission and strong support by the target-using communities will be necessary. In particular, an appropriate funding instrument has to be found and applied, enabling ANITA to develop from an initiative employed by the interested parties to a real coordination platform.
\end{abstract}

\section{INTRODUCTION}

Over the past 20 years, we observed an increasing demand on precise, accurate and reliable experimental data in various scientific research fields like nuclear astrophysics, basic nuclear physics, nuclear energy, nuclear medicine, geoscience, super-heavy element research and others. This interdisciplinary character (see Figure 1) of both basic and applied research in the field of nuclear science has been addressed within the EURATOM-funded project CHANDA [1] in work package WP2, dedicated to cross cutting with other research fields. As an outcome of CHANDA WP2, the need for well-characterized nuclear targets [2] became evident, and it is clearly visible that isotope and target preparation issues play a central role in any of these scientific fields. Since the production of high quality samples and their characterization requires high quality, expensive equipment, not many laboratories can afford having all necessary devices and a network allowing the development of a multidisciplinary collaboration in the field of target preparation science is emerging. However, at the moment there is no adequate funding instrument 
available within the European Union's framework program supporting such collaboration, beside the nuclear energy sector which is separately funded by the EURATOM program.

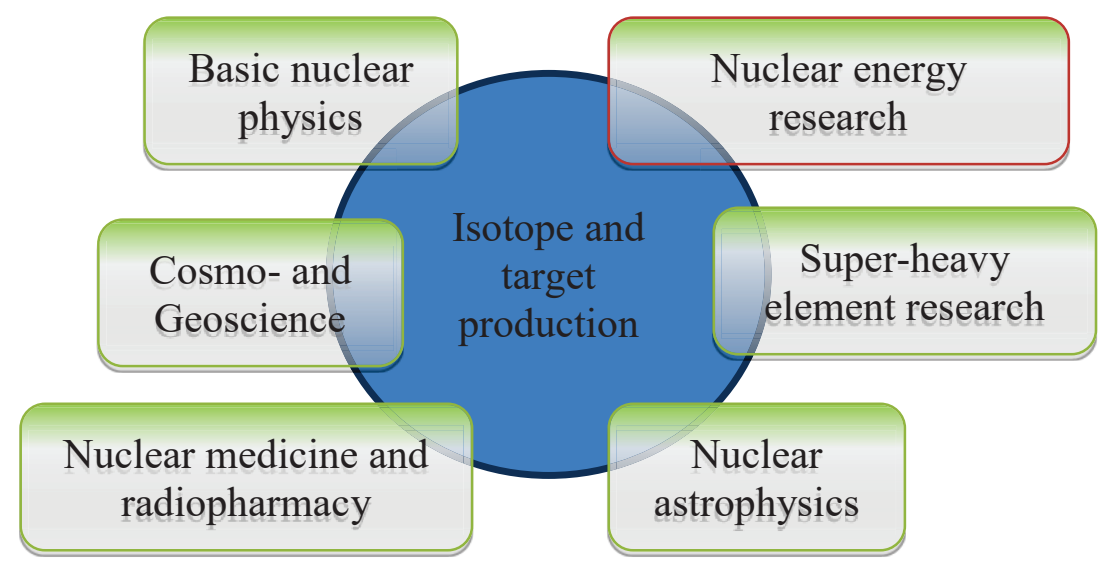

Fig. 1. Sketch of the synergies between different scientific and applied research fields and isotope and target production sites.

At present, several large-scale facilities run their own dedicated target laboratories. These target producers work mainly individually or collaborate with others on a very limited network level, even in the frame of pure nuclear energy research programs. Links to other research areas are missing or are only weakly established. Sharing of resources for isotope production and target preparation practically does not take place; coordination of requests is mostly left to the users. Often enough, users even do not include target production costs in their experimental budget and/or target delivering timeline in their schedule. Hence, the collaboration with the users in order to manufacture tailored samples or targets for their specific application has to be improved urgently. Owing to this situation, the development of an appropriate network according to the special demands of the user communities as well as a global coordination of the capabilities of the target laboratories in Europe is mandatory in order to optimize the use of the very limited resources for future ground-breaking experiments in the field of nuclear sciences. A first attempt to meet this urgent need was undertaken in the frame of the CHANDA project in form of a special work package: "Coordination of the development of a network for nuclear target preparation and characterization (WP3)", which is, however, restricted to nuclear energy related nuclear data research. A list of isotope production and target manufacturing sites has been generated, and support could be given to selected target manufacture assignments. A dedicated workshop held in November 2015 at PSI (CHANDA - workshop on target preparation - the needs and the possibilities [3]) identified the development of a target preparation and characterization network as a core requirement in experimental nuclear science. Similar statements were made at the $\mathrm{NuPeCC}$ astrophysics town meeting in February 2016 [4]. The topic "sample and target preparation" will be included as a key issue into the NuPeCC Long Range Plan to be launched in 2017.

\section{ANITA - AN ATTEMPT TO ESTABLISH A EUROPEAN SAMPLE AND TARGET PREPARATION NETWORK}

Due to the high expenditure, not every target laboratory can process every isotope or sample, and not every target laboratory has every instrument for target characterization available. Therefore, tasks for production as well as for characterization have to be coordinated and distributed between the facilities in Europe. To fulfill all the requirements to produce highest quality targets for front-end experiments, we need a completely new level of collaboration between target producers, sample deliverers and experimenters, covering not only network activities and mutual access to target production facilities, instruments and knowledge, but also concerted efforts for new developments in target production and characterization.

Searching for the solution of the above listed problems, and having the CHANDA WP3 experience, the following six target producing laboratories from institutions of four different countries started an initiative called ANITA to build up an appropriate network: 
- $\quad$ Target chemistry group (Laboratory of Radiochemistry, Nuclear Energy and Safety Research Department) at Paul Scherrer Institute Villigen (PSI), Switzerland,

- Target preparation laboratory (Directorate for Nuclear Safety and Security) at European Commission, Joint Research Centre Geel (EC-JRC.G),

- Target laboratory (Heavy Ion Laboratory) at the University of Warsaw, HIL-UW, Poland,

- Target laboratory (Institut für Kernchemie) at the Johannes Gutenberg-Universität in Mainz, (JGU) Germany,

- Target laboratory at the Helmholtz Centre for Heavy Ion Research Darmstadt (GSI), Germany,

- Target laboratory at Grand Accélérateur National d’Ions Lourds; Caen (GANIL), France.

The global aim of ANITA is to establish an overarching research infrastructure service for target production and to develop a tight cooperation between the target laboratories in Europe in order to transfer the knowledge and improve the production technique of well-characterized samples/targets. Moreover, the interaction of the target laboratories with the research teams using these targets shall be triggered and/or intensified to deliver targets bestsuited for the envisaged experiments.

The following objectives are envisaged:

- Identification and promotion of synergies with other target producers

- Establishing a network between the presently leading European infrastructures for isotope and target production

- Development of an open access data base on isotope production facilities and target manufacturers

- Coordination of targets requests, sharing of capacities, distribution of demands

- Transnational visits of scientists and especially students and Postdoc for knowledge exchange and training

- Development of new, innovative equipment for specific applications, accessible for the entire community

- Basic research on target preparation methods and characterization techniques; development of new, improved techniques

- Acquisition of new partners and broadening the network, also outside Europe

- $\quad$ Networking with the user communities.

The key activities envisaged in the future are fourfold:

\section{Network of target laboratories to share resources}

An overview on target production and characterization possibilities is one of the preconditions for an optimized coordination of all activities in the field. An open access electronic platform for all ANITA partners, collaborators and users as well as any other interested party is desired to provide the necessary information. This data base shall also contain information on availability of re-useable targets, their characteristics, available transport containers including their certificates and data on isotope production sites, storage places and commercial deliverers. Moreover, we need detailed descriptions of the possibilities for target characterization of collaborating target laboratories in Europe and world-wide. Target request lists are a further pre-requisite to coordinate target production and task distribution.

In many cases, the production routes and the separation and purification techniques already influence the quality of the final target. Target laboratories should, therefore, also tightly collaborate with isotope producers. Prediscussions on requirements for isotope production could help to lower the efforts for expensive purification steps and also help to find cost-effective radiochemical transformations. In some cases, the selection of a suitable production route is the key parameter for a successful experiment.

\section{Networking with the users to produce tailored targets}

The specification of a target is given by the requirements coming from the underlying physics (nuclear reactions, unwanted by-products), the possibilities of detection of the studied product and the specific configuration of the experimental setup. Front-end experiments in the field of nuclear data are not uniform. Every experiment is unique and the parameters are differing. The applied techniques are especially designed for the envisaged experiment and are far away from standard techniques. Consequently, also the target is to a large extent unique. A very tight contact with experimenters as well as the knowledge of the nuclear reaction to be studied, the detector properties and nearly any other boundary condition will contribute to the improvement of the target quality and, thus, to the outcome of the experiment. This goal can be reached by regular bilateral meetings of the target laboratory in charge and the experimenter on the one side and general user meetings on a more global level, where target producers and 
experimenters inform each other on new developments, successful scientific applications and future plans on the other side.

Trans-national access and support of young researchers

Exchange of scientists between the involved target laboratories is one of the main focuses. This concerns the gaining of knowledge on new or existing methods and technologies for target preparation and characterization. The network should give open access to a large number of end-users even if they are not partners or collaborators of the network.

Special attention should be paid to the training and education of PhD students and PostDocs. Young researchers and fellows shall have the opportunity for long-term visits at other institutes to broaden their knowledge and learn techniques which are not available at their home institutions. This requires also a very tight collaboration with Universities and colleges for supporting their careers including summer schools and training courses.

\section{Joint research activities}

Basic investigations and the development of new, innovative techniques in order to broaden the knowledge on target production and characterization are necessary to provide high-quality services for users and to deliver targets meeting the requirements of the envisaged experiments. An elaborated method and instrument development plan within the European Community for a cost-effective use of resources is mandatory.

\section{CONCLUSION}

The urgent demand of optimization and coordination of target producer's activities is obvious. A dedicated network combining the demands of users/experimenters and the needs of target producing facilities as well as fostering the collaboration between the target laboratories has to be established as fast as possible. However, due to the broad variety of the application fields, the identification and exploitation of an adequate funding instrument of this unique kind of collaboration remains currently the fundamental problem.

The ANITA initiative shall trigger the development of an international target-related collaboration. There is urgent need to broaden the community and give access to information and infrastructures available world-wide. In this respect, the International Nuclear Target Development Society (INTDS) is of particular importance as a wellknown, already existing international platform. With its bi-annual conference series, it is an ideal forum for maintaining existing and establishing new contacts, to identify synergies and optimize the entire target preparation business.

\section{ACKNOWLEDGEMENTS}

The work has partly been funded by CHANDA (grant agreement No FP7 - Fission-2013 - 605203).

\section{REFERENCES}

[1] CHANDA (solving CHAllenges in Nuclear Data), Project ID: 605203, http://cordis.europa.eu/project/rcn/110083 en.html

[2] B. Jurado, G. Barreau, C.-O. Bacri, Nucl. Instrum. Meth.Phys. Res. A613 (2010) 343-346

[3] CHANDA - workshop on target preparation - the needs and the possibilities, Villigen (Switzerland), 23.-25.11.2015, http://indico.psi.ch/conferenceDisplay.py?confId $=3671$

[4] NuPECC Long Range Plan Town Meeting,Darmstadt, January 11-13, 2017, http://indico.gsi.de/conferenceDisplay.py?confld=5177 